\title{
Palladium-catalyzed dehalogenation of benzofuran bromides
}

Wang Zunyuan, Ma Zhen, Yang Yewei, Liang Meihao, Huang Wenhai, Shen Zhengrong*

Zhejiang Academy of Medical Sciences, Hangzhou, Zhejiang, China 310013

Email: wzuny@163.com, Shenzr601@163.com

Table of Contents

I. Experimental Section ....................................S1-S3

II. Copies of ${ }^{1} \mathrm{H}$ and ${ }^{13} \mathrm{C}$ NMR spetra........................S4-S10 


\section{$\underline{\text { I. Experimental Section }}$}

\section{General}

All reactions were carried out using oven-dried glassware. $\operatorname{Pd}(\mathrm{OAc})_{2}$ and ligands a-e were purchased from Aldrich and was used without further purification. Potassium phosphate $\left(\mathrm{K}_{3} \mathrm{PO}_{4}\right)$ was purchased from Aldrich and was finely ground prior to use. Toluene and THF were purchased from Hangzhou Huipu Chemical Industry Instrument Co., Ltd, which were distilled from calcium hydride $\left(\mathrm{CaH}_{2}\right)$ immediately prior to use. All other reagents were commercially available and were used without further purification.

All reactions were monitored by TLC with Merck silica gel coated plates (60F254, Qingdao, China) using UV-light $(254 \mathrm{~nm})$. Column chromatography was carried out using Silica gel (HG/T2354-92) purchased from Qingdao. Melting points were determined with an X-4 apparatus and were uncorrected. Infrared spectra (IR) were measured on a Bruker Vector22 spectrometer. ${ }^{1} \mathrm{H}-\mathrm{NMR}(400 \mathrm{MHz})$ and ${ }^{13} \mathrm{C}-\mathrm{NMR}(100 \mathrm{MHz})$ spectra were recorded on a Bruker Avance spectrometer using tetramethylsilane (TMS) as internal standard. Chemical shifts $(\delta)$ are reported in ppm, and coupling constants $(J)$ are in Hz. Mass spectra (electron ionization (EI), $70 \mathrm{eV}$ ) were recorded on an Agilent 5975 inert mass selective detector. High resolution (HR) mass spectra (EI, $70 \mathrm{eV}$ ) were obtained by the corresponding service at Shanghai Institute of Organic Chemistry, Chinese Academy of Sciences using a Waters Micromass GCT apparatus.

\section{General Procedure for the Preparation of Brominated Substrates}

The brominated products were synthesized as followed. 27-30, and 40-42. To a solution of the requisite benzofuran (1 equiv) in THF $(0.4 \mathrm{M})$ at room temperature under argon was added NBS 
(1.2 equiv) in THF ( $0.35 \mathrm{M})$. After being stirred at room temprature in the absence of light for $3 \mathrm{hr}$, the reaction was quenched at room temperature with a saturated aqueous solution of $\mathrm{NaHCO}_{3}$, extracted three times with ethyl acetate. The combined organic layers were washed with a solution of sodium thiosulfate $12 \% \mathrm{w} / \mathrm{v}$ and brine, dried over $\mathrm{MgSO}_{4}$, filtered, and concentrated under reduced pressure. The crude product was purified by flash chromatography over silica gel to afford the desired product.

\section{3-Bromo-2-butyl-5-methanesulfonylaminobenzofuran}

White solid, mp $100-102^{\circ} \mathrm{C} .{ }^{1} \mathrm{H}-\mathrm{NMR}\left(400 \mathrm{MHz}, \mathrm{CDCl}_{3}\right) \delta: 7.53(1 \mathrm{H}, \mathrm{d}, \mathrm{J}=5.2 \mathrm{~Hz}), 7.39(1 \mathrm{H}, \mathrm{d}$, $\mathrm{J}=5 \mathrm{~Hz}), 6.64(1 \mathrm{H}, \mathrm{s}, \mathrm{br}, \mathrm{NH}), 6.43(1 \mathrm{H}, \mathrm{s}), 2.97(3 \mathrm{H}, \mathrm{s}), 2.77-2.81(2 \mathrm{H}, \mathrm{m}), 1.77-1.73(2 \mathrm{H}, \mathrm{m})$,

1.45-1.41(2H, m), 0.99-0.96(3H, m) . ${ }^{13} \mathrm{C}-\mathrm{NMR}\left(100 \mathrm{MHz}, \mathrm{CDCl}_{3}\right) \delta: 162.3,152.1,131.2,129.5$ 120.2, 110.8, 107.8, 102.5, 39.6, 29.6, 28.2, 22.2, 13.8. MS(EI)m/z(\%): 347(M+1), 345, 268, 266, 145. HR-MS(EI) Calcd for $\mathrm{C}_{13} \mathrm{H}_{16} \mathrm{BrNO}_{3} \mathrm{SNa}$, [M+Na] $]^{+}$: 367.9932, Found:367.9933.

\section{3-Bromo-6-methoxy-2-(4-methoxyphenyl)benzofuran}

White solid, mp $75-77^{\circ} \mathrm{C} .{ }^{1} \mathrm{H}-\mathrm{NMR}\left(400 \mathrm{MHz}, \mathrm{CDCl}_{3}\right) \delta: 8.32(2 \mathrm{H}, \mathrm{d}, \mathrm{J}=5.5 \mathrm{~Hz}), 7.64(1 \mathrm{H}, \mathrm{d}$, $\mathrm{J}=5 \mathrm{~Hz}), 7.28(2 \mathrm{H}, \mathrm{m}), 7.25(1 \mathrm{H}, \mathrm{s}), 717-7.20(1 \mathrm{H}, \mathrm{m}), 4.12(6 \mathrm{H}, \mathrm{s}) .{ }^{13} \mathrm{C}-\mathrm{NMR}\left(100 \mathrm{MHz}, \mathrm{CDCl}_{3}\right) \delta$ : $159.8,158.7,153.9,149.8,127.8,123.2,122.5,119.5,114.0,112.3,95.8,92.1,55.8,55.4$ $\operatorname{MS}(\mathrm{EI}) \mathrm{m} / \mathrm{z}(\%): 334\left(\mathrm{M}^{+}+2\right), 332\left(\mathrm{M}^{+}\right), 319,317,167,139 . \mathrm{HR}-\mathrm{MS}(\mathrm{EI})$ Calcd for $\mathrm{C}_{16} \mathrm{H}_{14} \mathrm{BrO}_{3}$ $[\mathrm{M}+\mathrm{H}]^{+}:$333.0126, Found: 333.0119 .

\section{3-Bromo-2-(3,4-dimethoxyphenyl)benzofuran}

White solid, mp $90-92^{\circ} \mathrm{C} .{ }^{1} \mathrm{H}-\mathrm{NMR}\left(400 \mathrm{MHz}, \mathrm{CDCl}_{3}\right) \delta:$ 7.77-7.80(1H, dd, J=5.2Hz), 7.71 $(1 \mathrm{H}, \mathrm{d}, \mathrm{J}=1 \mathrm{~Hz}), 7.53-7.55(1 \mathrm{H}, \mathrm{m}), 7.48-7.51(1 \mathrm{H}, \mathrm{m}), 7.29-7.36(2 \mathrm{H}, \mathrm{m}), 6.98(1 \mathrm{H}, \mathrm{d}, \mathrm{J}=5.2 \mathrm{~Hz})$ 4.0(3H,s), 3.95(3H, s). ${ }^{13} \mathrm{C}-\mathrm{NMR}\left(100 \mathrm{MHz}, \mathrm{CDCl}_{3}\right) \delta: 152.9,150.3,149.8,148.9,129.8,125.2$ 
$123.4,122.3,120.0,119.6,111.1,111.0,109.7,92.5,56.0 . \mathrm{MS}(\mathrm{EI}) \mathrm{m} / \mathrm{z}(\%): 334\left(\mathrm{M}^{+}+2\right), 332\left(\mathrm{M}^{+}\right)$,

319, 317, 210, 139, 99, 56. HR-MS(EI) Calcd for $\mathrm{C}_{16} \mathrm{H}_{14} \mathrm{BrO}_{3},[\mathrm{M}+\mathrm{H}]^{+}:$333.0126, Found: 333.0119.

\section{3-Bromo-2-(3,4-dimethoxyphenyl)-6-methoxybenzofuran}

White solid, mp 105-107 ${ }^{\circ}$. ${ }^{1} \mathrm{H}-\mathrm{NMR}\left(400 \mathrm{MHz}, \mathrm{CDCl}_{3}\right) \delta: 7.73(1 \mathrm{H}$, dd, J=5 Hz), 7.67(1H, d, $\mathrm{J}=1 \mathrm{~Hz}), 7.39(1 \mathrm{H}, \mathrm{d}, \mathrm{J}=5.5 \mathrm{~Hz}), 7.05(1 \mathrm{H}, \mathrm{d}, \mathrm{J}=1.2 \mathrm{~Hz}), 6.98(1 \mathrm{H}, \mathrm{d}, \mathrm{J}=5.2 \mathrm{~Hz}), 6.93-6.96(1 \mathrm{H}, \mathrm{m})$, 4.12(9H, s). ${ }^{13} \mathrm{C}-\mathrm{NMR}\left(100 \mathrm{MHz}, \mathrm{CDCl}_{3}\right) \delta: 158.8,153.9,149.4,148.9,123.2,122.6,119.8,119.5$, 112.4 111.1, 109.4, 95.8, 92.3, 56.0, 55.8. MS(EI)m/z(\%): 364(M $\left.{ }^{+}+2\right), 362\left(\mathrm{M}^{+}\right), 349,347,240$, 225. HR-MS(EI) Calcd for $\mathrm{C}_{17} \mathrm{H}_{15} \mathrm{BrO}_{4} \mathrm{Na}$, [M+Na] $]^{+}: 385.0051$, Found:385.0053.

\section{3-Bromo-2-(4-bromophenyl)benzofuran}

White solid, mp 191-193 ${ }^{\circ} \mathrm{C} .{ }^{1} \mathrm{H}-\mathrm{NMR}\left(400 \mathrm{MHz}, \mathrm{CDCl}_{3}\right) \delta: 7.53-7.59(6 \mathrm{H}, \mathrm{m}), 7.37(1 \mathrm{H}, \mathrm{d}$, $\mathrm{J}=5.2 \mathrm{~Hz}), 7.29-7.33(1 \mathrm{H}, \mathrm{m}) .{ }^{13} \mathrm{C}-\mathrm{NMR}\left(100 \mathrm{MHz}, \mathrm{CDCl}_{3}\right) \delta: 160.3,153.5,140.0,133.6,131.7$, 130.1, 128.0, 127.2, 124.7, 123.2, 119.5, 113.6. $\mathrm{MS}(\mathrm{EI}) \mathrm{m} / \mathrm{z}(\%): 302\left(\mathrm{M}^{+}+2\right), 300\left(\mathrm{M}^{+}\right), 274,272$,

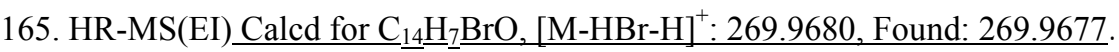

\section{3-Bromo-2-(4-bromophenyl)-6-methoxy benzofuran}

White solid, mp 106-108 ${ }^{\circ} \mathrm{C} .{ }^{1} \mathrm{H}-\mathrm{NMR}\left(400 \mathrm{MHz}, \mathrm{CDCl}_{3}\right) \delta: 7.99(1 \mathrm{H}, \mathrm{d}, \mathrm{J}=3 \mathrm{~Hz}), 7.66(1 \mathrm{H}, \mathrm{d}$, $\mathrm{J}=5 \mathrm{~Hz}), 7.59(1 \mathrm{H}, \mathrm{d}, \mathrm{J}=4.5 \mathrm{~Hz}), 7.54(1 \mathrm{H}, \mathrm{d}, \mathrm{J}=5.2 \mathrm{~Hz}), 7.40-7.45(1 \mathrm{H}, \mathrm{m}), 7.02-7.06(1 \mathrm{H}, \mathrm{m})$, 6.946.96(1H, m), 3.74(3H, s). ${ }^{13} \mathrm{C}-\mathrm{NMR}\left(100 \mathrm{MHz}, \mathrm{CDCl}_{3}\right) \delta: 159.3,158.3,154.1,131.9,131.8$, 127.6, 125.9, 121.1, 120.2, 112.9, 112.2, 101.8, 95.9, 95.7, 92.3, 55.8. $\mathrm{MS}(\mathrm{EI}) \mathrm{m} / \mathrm{z}(\%): 382\left(\mathrm{M}^{+}+2\right)$, $380\left(\mathrm{M}^{+}\right), 367,304,302,289,287,180,152,150$. HR-MS(EI) Calcd for $\mathrm{C}_{16} \mathrm{H}_{12} \mathrm{BrO}_{2}$, $[\mathrm{M}-\mathrm{HBr}-\mathrm{H}]^{+}: 315.0021$, Found: 315.0020. 


\section{General Procedure for the Pd-Catalyzed Dehalogenation of Benzofuran Halides}

An oven-dried screw-cap tube was charged with ligand (10 mol\%), $\operatorname{Pd}(\mathrm{OAc})_{2}(10 \mathrm{~mol} \%, 0.1$ $\mathrm{mmol}, 22.4 \mathrm{mg}), \mathrm{K}_{3} \mathrm{PO}_{4}(2.0 \mathrm{mmol}, 424 \mathrm{mg})$, and the aryl halide $(1.0 \mathrm{mmol})$. The tube was capped with a septum and under a positive pressure of nitrogen, toluene $(2 \mathrm{~mL})$ was added via syringe through the septum, followed by a little of water $(100 \mathrm{uL} \underline{\mu \mathrm{L}, 5 \text { equiv }})$. The reaction flask was sealed and was placed in a pre-heated oil bath at 100 and stirred for 24 or $48 \mathrm{~h}$ (reaction times were not optimized). At this time the reaction mixture was allowed to cool to room temperature and was then filtered through a small pad of Celite, washed with diethyl ether or ethyl acetate (25 $\mathrm{mL}$ ) and concentrated under reduced pressure. The crude product was purified by flash chromatography on silica gel.

\section{2-Butyl-5-methanesulfonylaminobenzofuran}

White solid, mp $100-102^{\circ} \mathrm{C} .{ }^{1} \mathrm{H}-\mathrm{NMR}\left(400 \mathrm{MHz}, \mathrm{CDCl}_{3}\right) \delta:$ 7.53(1H, d, J=5.2 Hz), 7.39(1H, d, $\mathrm{J}=5 \mathrm{~Hz}), 6.64(1 \mathrm{H}, \mathrm{s}, \mathrm{br}, \mathrm{NH}), 6.43(1 \mathrm{H}, \mathrm{s}), 2.97(3 \mathrm{H}, \mathrm{s}), 2.77-2.81(2 \mathrm{H}, \mathrm{m}), 1.77-1.73(2 \mathrm{H}, \mathrm{m})$, 1.45-1.41(2H, m), 0.99-0.96(3H, m) . ${ }^{13} \mathrm{C}-\mathrm{NMR}\left(100 \mathrm{MHz}, \mathrm{CDCl}_{3}\right) \delta: 162.3,152.1,131.2,129.5$, 120.2, 110.8, 107.8, 102.5, 39.6, 29.6, 28.2, 22.2, 13.8. MS(EI)m/z(\%): 267(M+), 188, 145, 118.

All the other debrominated products and the NMR spectra were published in our early literature, and the reference was listed as below:

Zhang, P.; Yang, Y.; Zheng, X.; Huang, W.; Ma, Z.; Shen, Z. Chem. Pharm. Bull., 2012, 60, 270. 


\section{Copies of ${ }^{1} \mathrm{H}$ NMR and ${ }^{13} \mathrm{C}$ NMR spetra}
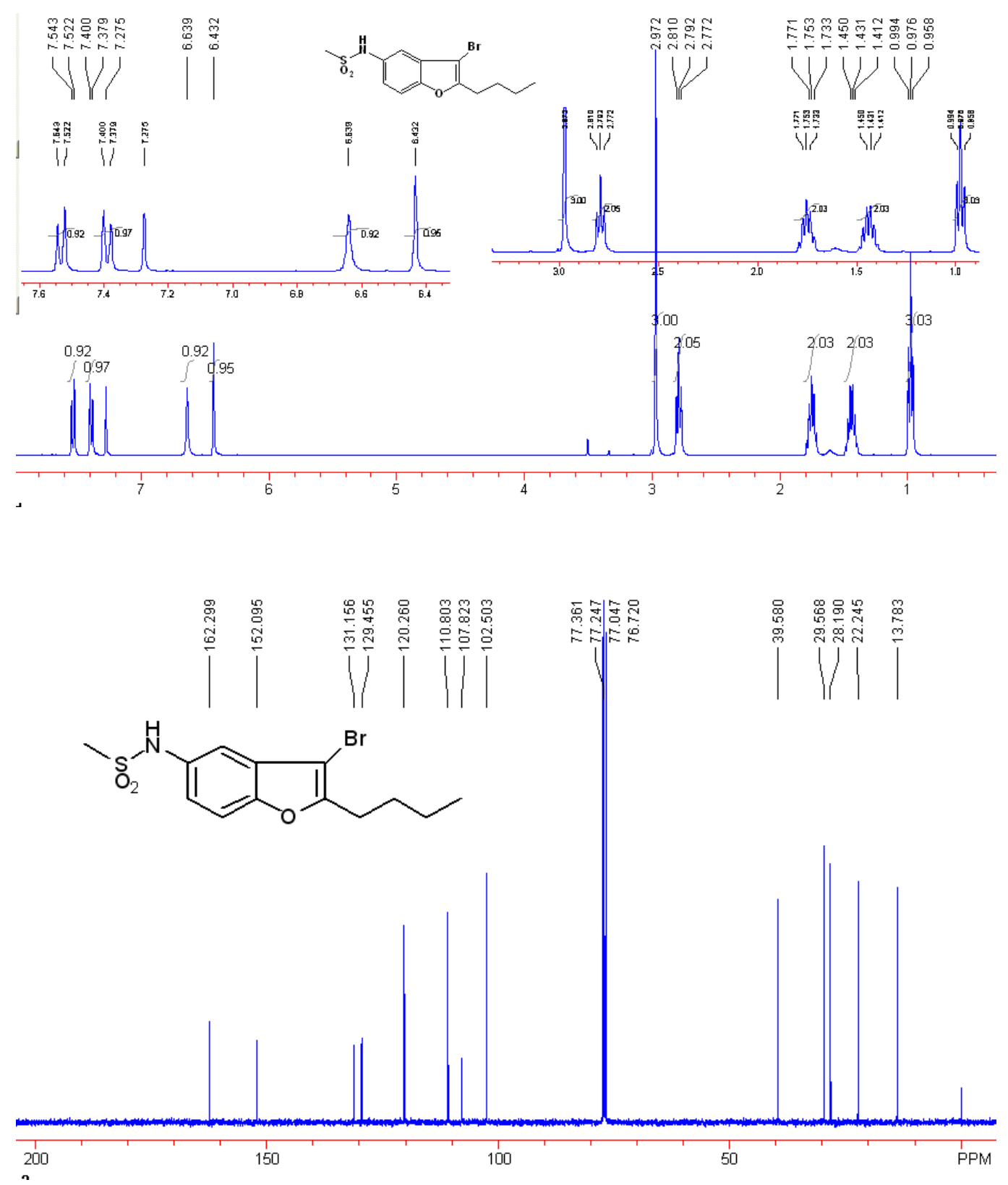

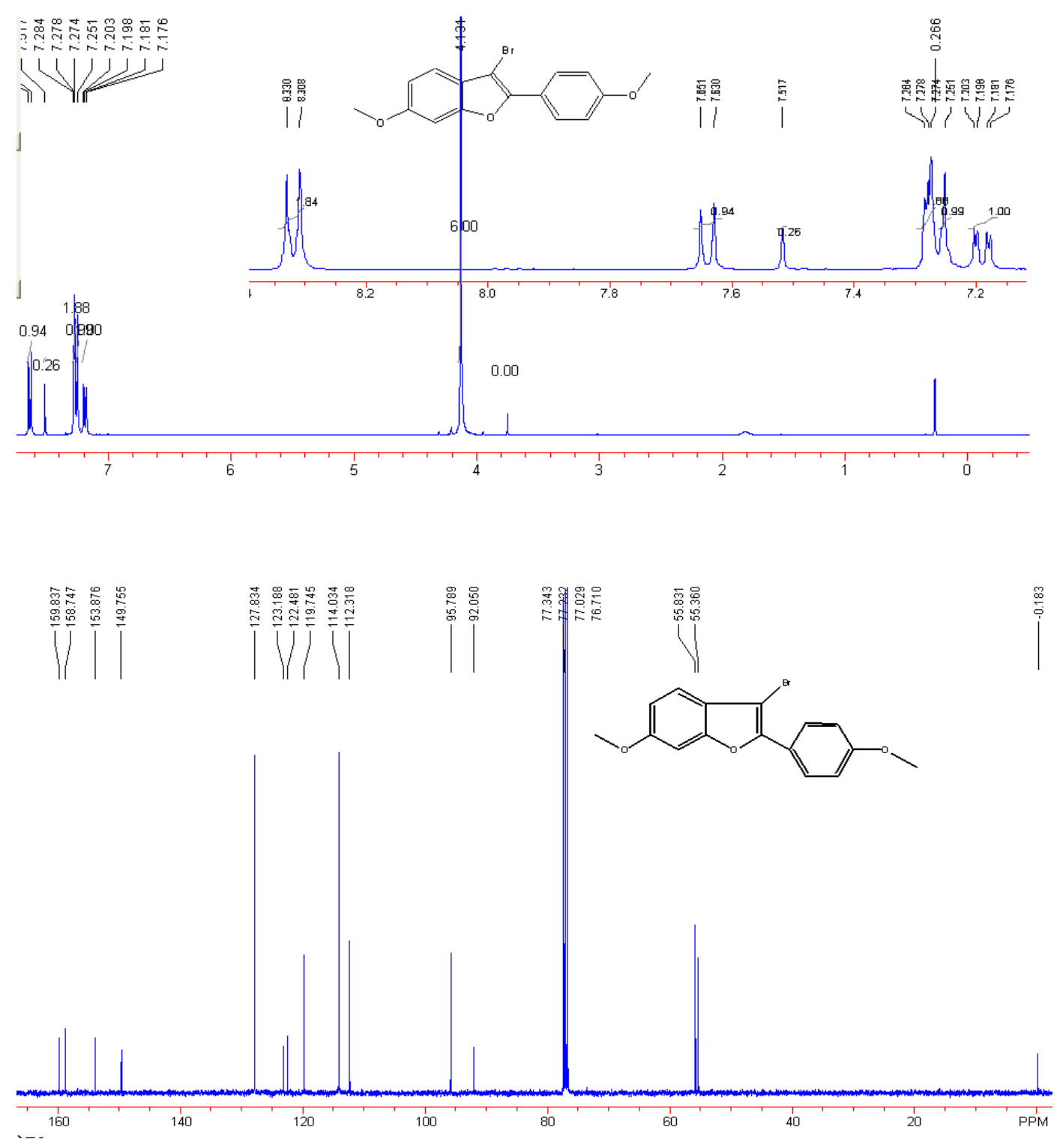

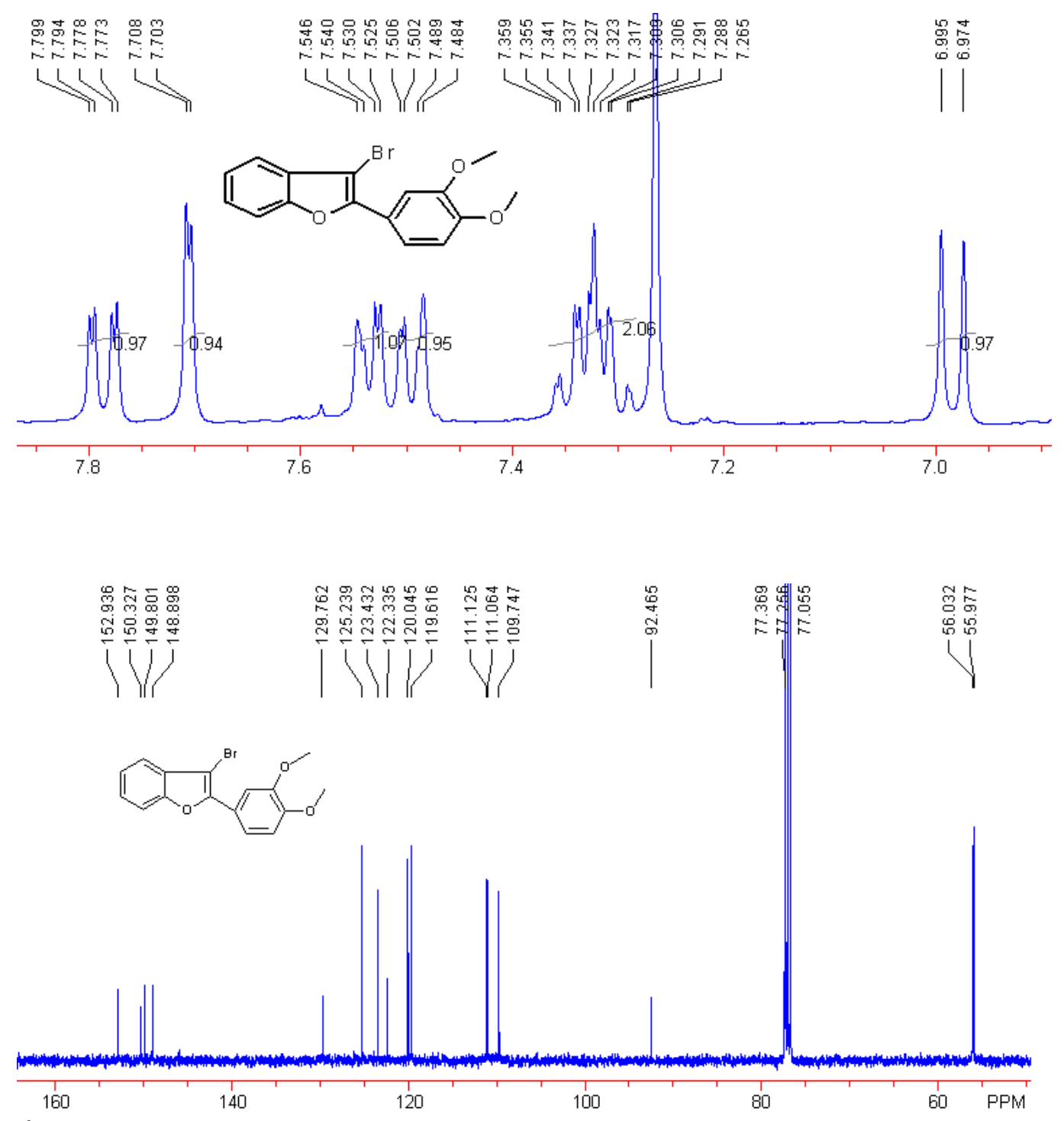

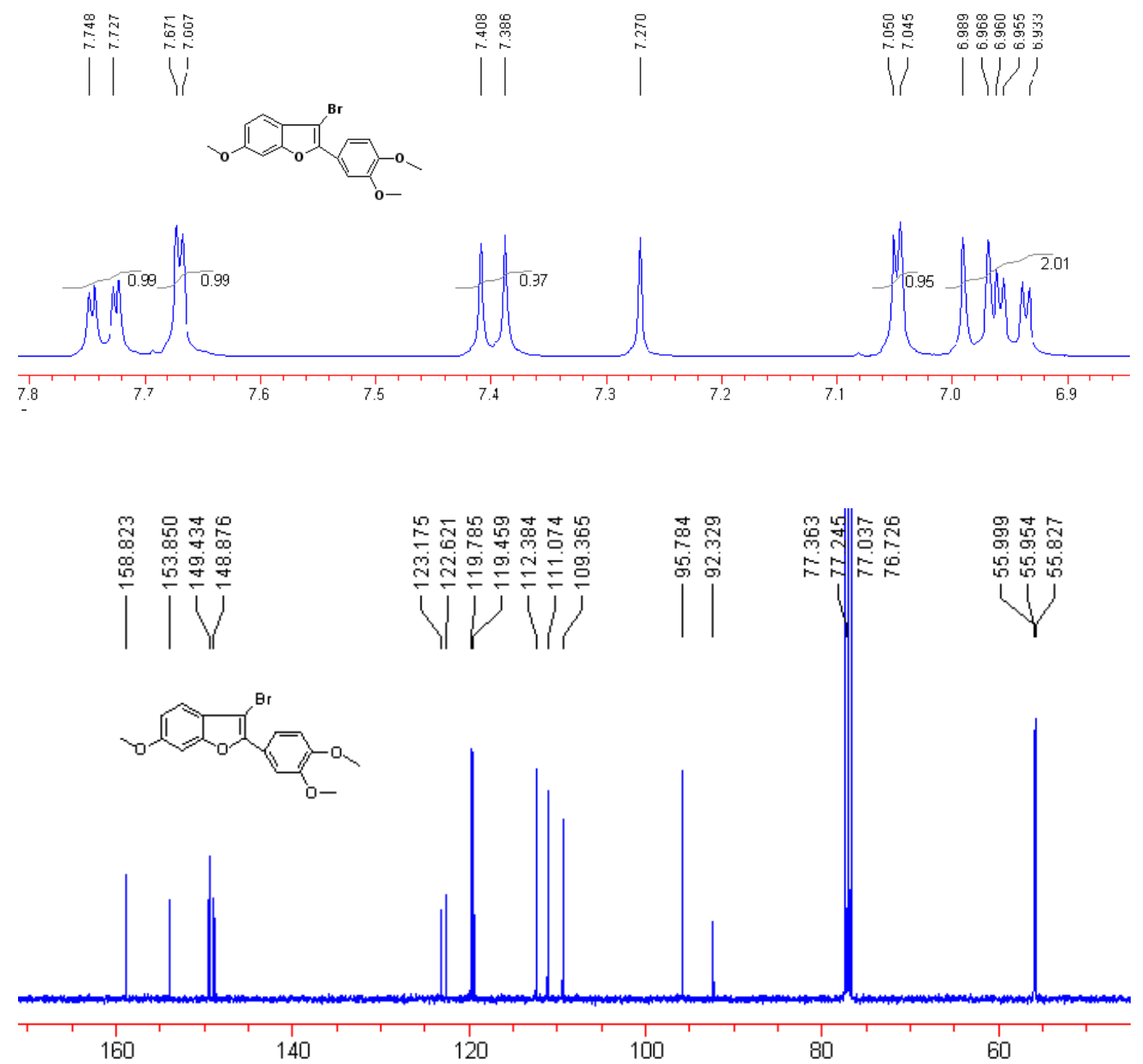

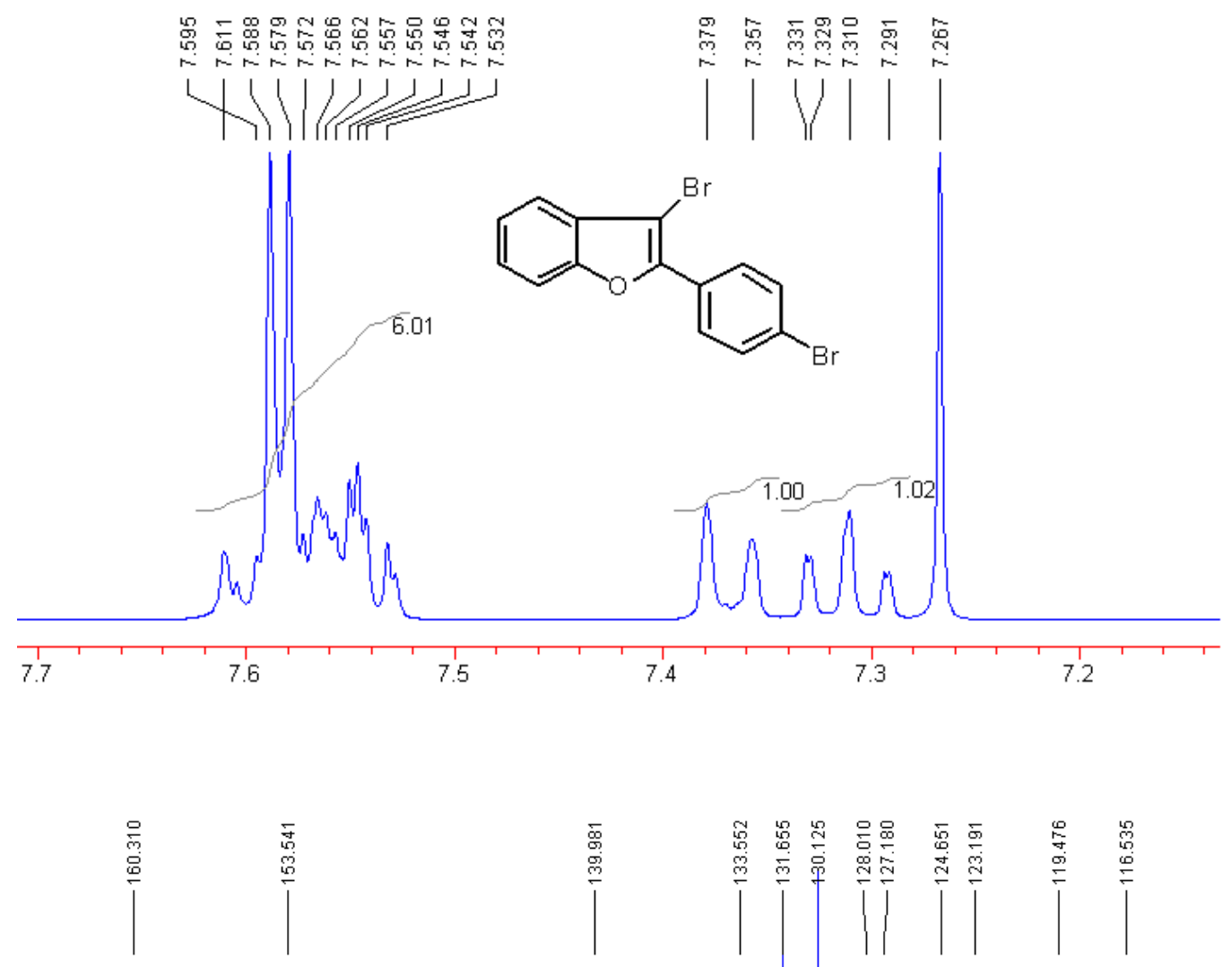<smiles>Brc1ccc(-c2oc3ccccc3c2Br)cc1</smiles>

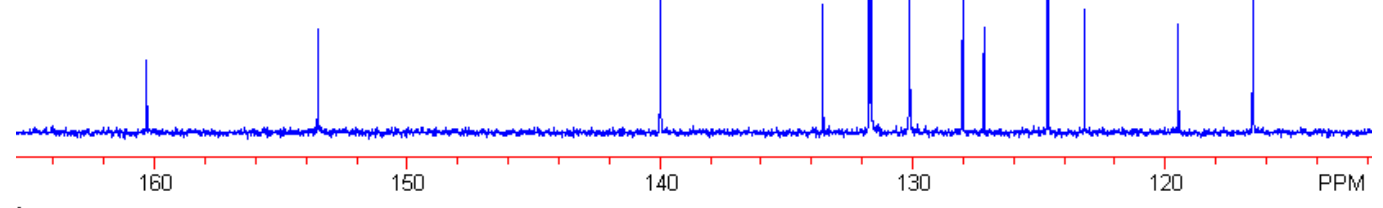



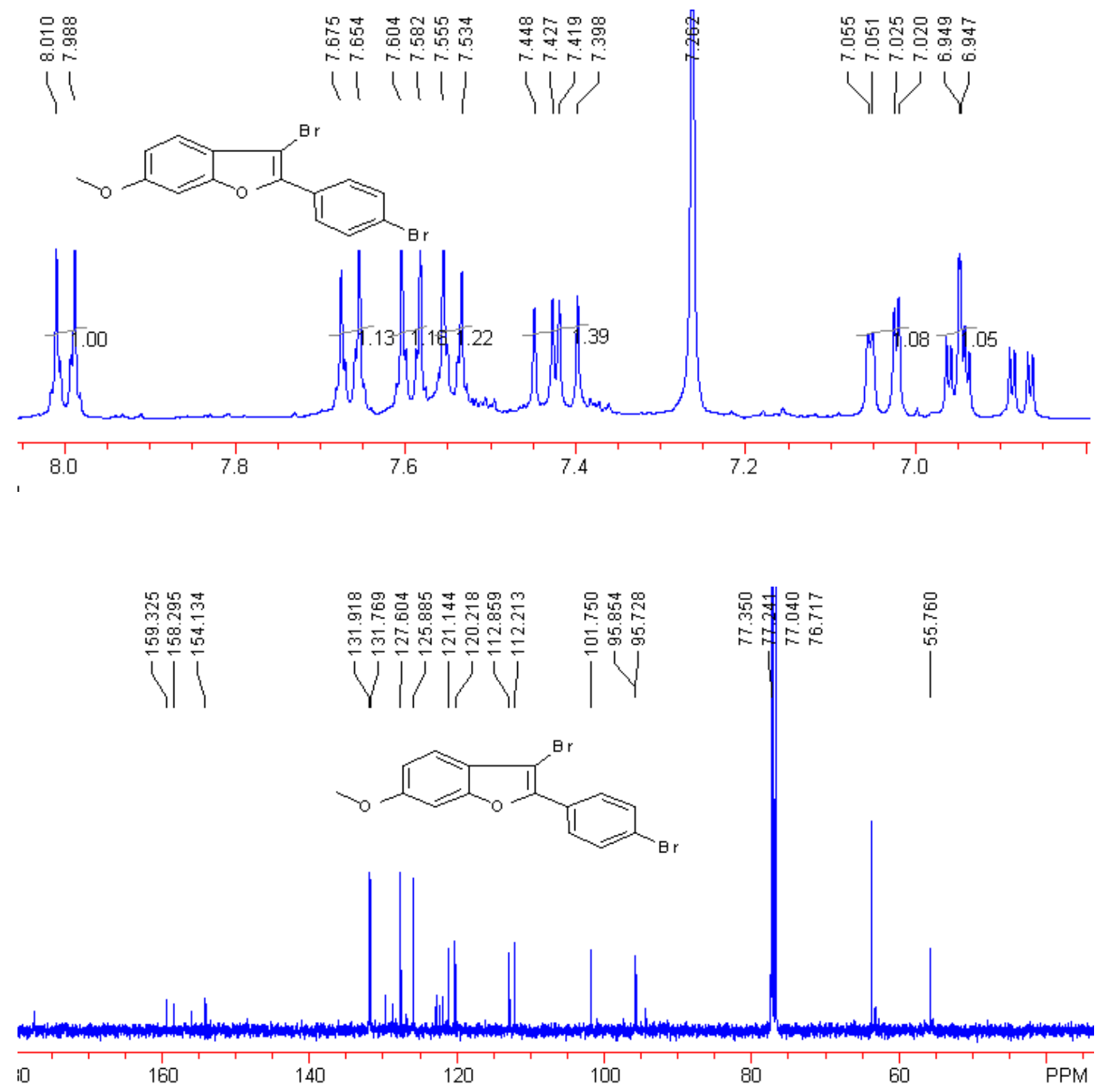